\title{
Status of p53 and p27krp in Iranian Patients With Oral Squamous Cell Carcinoma
}

\author{
Shahroo Etemad-Moghadam, ${ }^{1}$ Amanollah Keyhani, ${ }^{2}$ Kamran Yazdani, ${ }^{3}$ and Mojgan Alaed- \\ $\operatorname{dini}{ }^{1, *}$ \\ ${ }^{1}$ Dental Research Center, Dentistry Research Institute, Tehran University of Medical Sciences, Tehran, IR Iran \\ ${ }_{2}^{2}$ Meraj Cancer Institute, Tehran University of Medical Sciences, Tehran, IR Iran \\ ${ }^{3}$ Department of Epidemiology and Biostatistics, School of Public Health, Tehran University of Medical Sciences, Tehran, IR Iran \\ *Corresponding Author: Mojgan Alaeddini, Dental Research Center, Dentistry Research Institute, Tehran University of Medical Sciences, Tehran, IR Iran. Tel: +98-2188986677, \\ Fax: +98-2188986688, E-mail: malaeddini@yahoo.com
}

Received: April 8, 2014; Revised: July 21, 2014; Accepted: August 28, 2014

\begin{abstract}
Background:Alterations in p53 and p2 $7^{\mathrm{KIP} 1}$ have been documented as important events in the carcinogenesis of various cancers, but their prognostic role in oral squamous cell carcinoma (OSCC) remains controversial.

Objectives: The present investigation aimed to evaluate the clinicopathologic and prognostic significance of p53 and p2 $7^{\mathrm{KIP} 1}$ expression in a group of Iranian patients with OSCC.

Patients and Methods: In this analytical cross-sectional study, medical records of patients with primary OSCC, diagnosed from 1994 to 2004 were reviewed and 28 subjects were selected based on the inclusion/exclusion criteria. Immunohistochemical staining using monoclonal antibodies against $\mathrm{p} 53$ and $\mathrm{p} 27^{\mathrm{KIP1}}$ was performed on representative archival paraffin blocks. Demographic data along with information on $\mathrm{p} 53$ and p2 ${ }^{\mathrm{KIP} 1}$ expression, recurrence, and tumor grade was statistically analyzed using the Fischer exact test. Prognostic factors for overall survival were determined by Cox regression analysis $(\mathrm{P}<0.05)$.

Results: p53 and p27 $7^{\mathrm{KIP} 1}$ expression were found in $28.57 \%$ ( 8 positive versus 20 negative) and $67.85 \%$ (19 positive versus 9 negative) of OSCC cases, respectively. There was no significant association between these two proteins $(\mathrm{P}=0.371)$, and neither of them showed a significant relationship with the studied clinicopathologic variables $(\mathrm{P}>0.05)$. In survival analysis, only histopathologic differentiation (17 low and moderate, 11 poor) demonstrated a significant correlation with overall survival $(\mathrm{P}=0.048)$.

Conclusions: Despite the fact that abnormalities in p53 and p $27^{\mathrm{KIP} 1}$ may be involved in the development of OSCC, their clinical significance in the studied population seems limited. Further investigation on the combined p53/p2 $7^{\mathrm{KIP} 1}$ expression may be helpful in predicting the biologic behavior of this tumor.
\end{abstract}

Keywords: Survival; Squamous Cell Carcinoma; Tumor Suppressor Protein p53; Cyclin-Dependent Kinase Inhibitor p27

\section{Background}

Squamous cell carcinoma (SCC), the most common cancer of the oral cavity, is known to be associated with significant morbidity and mortality rates throughout the world. Considering the gradual increase in its prevalence and the relatively low 5 -year survival, which has improved poorly over the past years, the need for a general perception of the fundamental molecular mechanisms responsible for its formation and progression seems imperative $(1,2)$.

The combination and interaction of several aberrant molecular processes within the cell can lead to carcinogenesis. However in approximately all cases, underlying the neoplastic development is a disturbance in the balance between cell proliferation, differentiation, and death that mostly occur during progression through the cell cycle. Tumor cells have been shown to alter various pathways and cause abnormalities in cell cycleassociated gene products in order to achieve continu- ous proliferation and to remain in the cycle (3). P53 is a multifunctional protein with tumor suppressor and transcriptional activities. Following stress signals, its main effects on proliferation are exerted by controlling cell cycle transit principally at the G1/S checkpoint, resulting in temporary cell cycle arrest, differentiation, senescence, or apoptosis (4). In addition, p53 has been recently shown to affect a variety of cellular features and processes, including cellular metabolism, noncoding ribonucleic acids (RNAs), tumor cell invasion, and possibly "reprogramming of differentiated cells into induced pluripotent stem cells" (5). Because of this wide spectrum of biological functions it could be reasonably anticipated that p53 would be the most commonly targeted gene by human tumors in order to ensure their survival (6). The relationship between p53 expression and patient outcome is controversial in the published literature, especially in the head and neck region (7-9). 
Another cell cycle related factor is p27, a known universal Cyclin-dependent kinase (CDK) inhibitor, which can also function as a tumor suppressor gene and has been shown to regulate drug resistance in solid tumors, influence apoptosis, affect cell differentiation in some tissues, and protect a number of cell types against inflammatory injury (10). Previous studies have suggested a role for this molecule in carcinogenesis but reports on its relevance as a prognostic indicator have been inconsistent (9-15).

Despite the fact that p27 and p53 are mostly known to function independently and may not seem to cross paths during progression through the cell cycle (11), a number of investigations have shown that synchronous changes in the expression of these proteins may demonstrate a supplementary influence leading to increased proliferation and invasiveness $(11,14,16,17)$. Besides, there is evidence that these essential molecules may cross paths via interaction with related proteins and affect each other during cell cycle progression $(17,18)$.

\section{Objectives}

Deregulated cell proliferation is a fundamental event in carcinogenesis. Progression through the cell cycle lies at the heart of this event and therefore any alterations in its components may lead to the development of cancer (19). p53 tumor-suppressor and p27 ${ }^{\mathrm{KIP} 1}$ CDKinhibitor are known key factors in cell cycle regulation and provide valuable markers for evaluation in cancer patients. According to former investigations their prognostic role in oral SCC remains controversial and we were not able to find any previous reports on the expression of these markers in Iranian subjects with oral SCC $(8,9)$. The aim of the present study was to evaluate the status of p53 and p27 in a group of Iranian patients with oral SCC and to assess the correlation between these proteins.

\section{Patients and Methods}

\subsection{Samples}

In this survival study, medical records of patients treated at cancer institute, Tehran university of medical sciences and a number of private clinics in Tehran, Iran were retrospectively reviewed from 1994 to 2004 . It is noteworthy that Cancer Institute is a public university hospital and one of the main referral centers in Tehran that accepts patients from all over the country. Demographic data plus information regarding chief complaints of all subjects with biopsy-proven diagnoses of oral SCC were collected. Only primary tumor samples with available Hematoxylin and Eosin (H \& E) slides of the initial neoplasms were chosen and those with any of the following criteria were excluded from the study sample: SCC of the lip, incomplete medical and contact data, presence of a previously diagnosed cancer of any type and in any part of the body, history of radio/chemotherapy prior to the first surgical treatment and specific systemic, or any genetic predisposing factors to development of cancer (e.g. Plummer-Vinson syndrome, xeroderma pigmentosum, or Li-Fraumeni syndrome). All H\&E slides, obtained from the pathology archives of the respective clinics or hospital, were re-evaluated by two oral pathologists using a double-headed microscope (Olympus BX51) in order to confirm the initial diagnosis using the world health organization specifications (20). Also, the tumors were graded according to the criteria proposed by Bryne et al. (21) and disagreements were resolved by consensus. The protocol of this investigation was approved by the Ethics Committee of our University (code No: 840370). Written consents were obtained from all participants after explaining the study protocol and objectives. Personal information of all patients remained confidential and in order to avoid loss of specimens, only paraffin blocks with adequate amount of neoplastic tissues were selected and sectioning was kept to a minimum.

\subsection{Staining Procedure}

Immunohistochemical staining procedures were performed according to the instructions provided by the manufacturers. In brief, $4-\mu \mathrm{m}$ thick paraffin sections mounted on poly-L-lysine-coated glass slides were dewaxed in xylene, dehydrated, placed in $0.1 \mathrm{M}$ citrate buffer (PH: 0.6) and microwaved twice for 15 minutes followed by blocking of endogenous peroxidase activity with $0.3 \%$ hydrogen peroxide in methanol for half an hour. Incubation with monoclonal antibodies against p27 (IB4, Novocastra) and p53 (DO-7, Dako SA, Glostrup, Denmark) was carried out using dilutions of 1:20 and 1:50, respectively. All sections were then treated with avidin-biotin-peroxidase complex followed by immersion in diaminobenzidine- $\mathrm{H} 2 \mathrm{O} 2$ substrate (5 minutes, room temperature) for chromogen development and finally counterstained with hematoxylin. A known p53 positive oral SCC specimen and a section of human tonsil (for p27) were used as positive controls. Substitution of primary antibody with nonimmune serum, served as negative control.

\subsection{Immunohistochemical Analysis}

Labeling indices of all specimens were determined by two oral and maxillofacial pathologists using the abovementioned double-headed microscope without knowledge of their clinical features and outcomes. Any possible disagreements were resolved by consensus. A minimum of 300 cells in a total of 5 high power fields (HPFs) were counted and cases with $>10 \%$ nuclear staining of tumor cells were considered positive for p27 (12). P53 labeling index was calculated by assessing nuclear immunopositivity in 1000 neoplastic cells in 10 HPFs 
which was considered positive where there was $\geq 10 \%$ immunostaining (16).

\subsection{Follow up}

Recall visits were scheduled at 4-month intervals and all patients fulfilling the inclusion criteria were called to book appointments. If alive, they were required to visit their primary clinics where physical examination was performed by visual intraoral inspection using mouth mirrors on dental chairs equipped with standard light sources. Extraoral examination included palpation of neck node levels I through V, while the subjects were seated in a comfortable upright position. All examinations were done by a single operator specialized in oral and maxillofacial surgery based on the criteria proposed by Carlson et al. (22).

\subsection{Statistical Analysis}

Using SPSS.17 software, the Fischer exact test was employed to assess significant correlations between the categorical variables. Survival curves were plotted by the Kaplan-Meier method and compared using the log-rank test. Survival data were evaluated using Cox regression analyses and $\mathrm{P}<0.05$ was considered statistically significant.

\section{Results}

\subsection{Patients' Profile}

Based on the inclusion and exclusion criteria, 39 cases were selected for this study. Unfortunately, because of unobtainable paraffin blocks, including those with insufficient residual tumor and changes in contact information, 11 cases were lost at the initial stage of the investigation; leaving 28 samples for evaluation.

A total of $9(32.1 \%)$ men with a mean age of 54.4 years (range: 34 to $68 \mathrm{y}$ ) and 19 (67.9\%) women with a mean age of 58.39 years (range: 36 to $75 \mathrm{y}$ ) constituted our study population. There was no significant difference regarding the mean age between male and female subjects ( $P$ $=0.22$ ). The most common primary tumor site was the tongue (12 cases) followed by the gingiva ( 5 patients) and buccal mucosa (3 subjects).

In reviewing the clinical data of the study sample, we found the youngest patient to be a 34-year-old male whose chief complaint was of a white plaque on the tongue. The oldest subjects were two 75-year-old women, one of them initially attended the clinic/hospital for a large palpable mass involving the gingiva, base of the tongue and buccal mucosa. The other female subject complained of dentalgia, which finally led to a diagnosis of palatal SCC through clinical examination. All three died of their disease, 9, 10 and 11 months after the initial diagnosis, respectively.

Our cases were followed for a mean period of 59.71 months and a median of 55.50 months (range: 4 to 123 mo). At the end of the follow-up period, 9 (32.1\%) patients died because of their disease and of the 19 (67.9\%) censored subjects, 4 (21.1\%) demonstrated recurrence and 15 (78.9) were free of tumor. Considering the entire sample, tumor recurrence was found in 10 (35.7\%) cases, of them 1 was local, 4 were regional and 4 had metastasis to the neck and submandibular lymph nodes.

Because of the limited number of well-differentiated neoplasms, grades 1 and 2 tumors were combined (60.7\%) and compared with grade 3 (39.3\%) SCCs.

\subsection{Immunohistochemical Expression of p27 and p53}

We observed p53 and p27 immunopositivity in 8 (28.5\%) and 19 (67.8\%) patients, respectively (Figure 1). Both were confined mostly to the neoplastic nuclei. Overexpression of p53 was found in 4 women and 4 men, while p27 overexpression was found in 4 female and 15 male subjects. Significant differences between men and women with regard to p53 $(\mathrm{P}=0.37)$ and p27 $(\mathrm{P}=0.09)$ were not observed. In total, 4 patients were positive and 5 subjects were negative for both markers. Profiles of $\mathrm{p} 53^{+} / \mathrm{p} 27^{-}$and $\mathrm{p} 53^{-} / \mathrm{p} 27^{+}$were found in 4 and 15 cases, respectively. Comparing the expression of p53 and p27, did not reveal a significant association between these biomarkers $(P=0.37)$; however, there was a trend toward p53 negativity and p27 positivity. A significant correlation between the expression of these proteins and variables of age, gender, local recurrence, or pathologic differentiation was not observed $(P>0.05)$.

\subsection{Survival Analysis}

Overall survival (mean: 89; median: 97 months) was described as life or death at the time of last follow up. No loss to follow up was encountered in the present study. Patients with negative and positive p27 expression had mean survivals of 76.11 (95\% CI; $51.78-100.43$ months) and 87.60 months (95\% CI; 66.9-108.2 months), respectively (Figure 2). A mean survival of 90.63 months (95\% CI; 70.2 - 111.0 months) was found in p53 negative subjects which was calculated as 83.12 months (95\% CI; 49.8 - 116.4 months) in those with positive p53 (Figure 3). Univariable analysis of clinicopathologic characteristics showed no significant association between overall survival and the studied variables (age, gender, local recurrence, and p27/p53 expression). As observed in Table 1, only pathologic differentiation emerged as an independent prognostic factor $(\mathrm{P}=0.04)$. According to Cox regression analysis, none of these parameters had a significant impact on survival. 


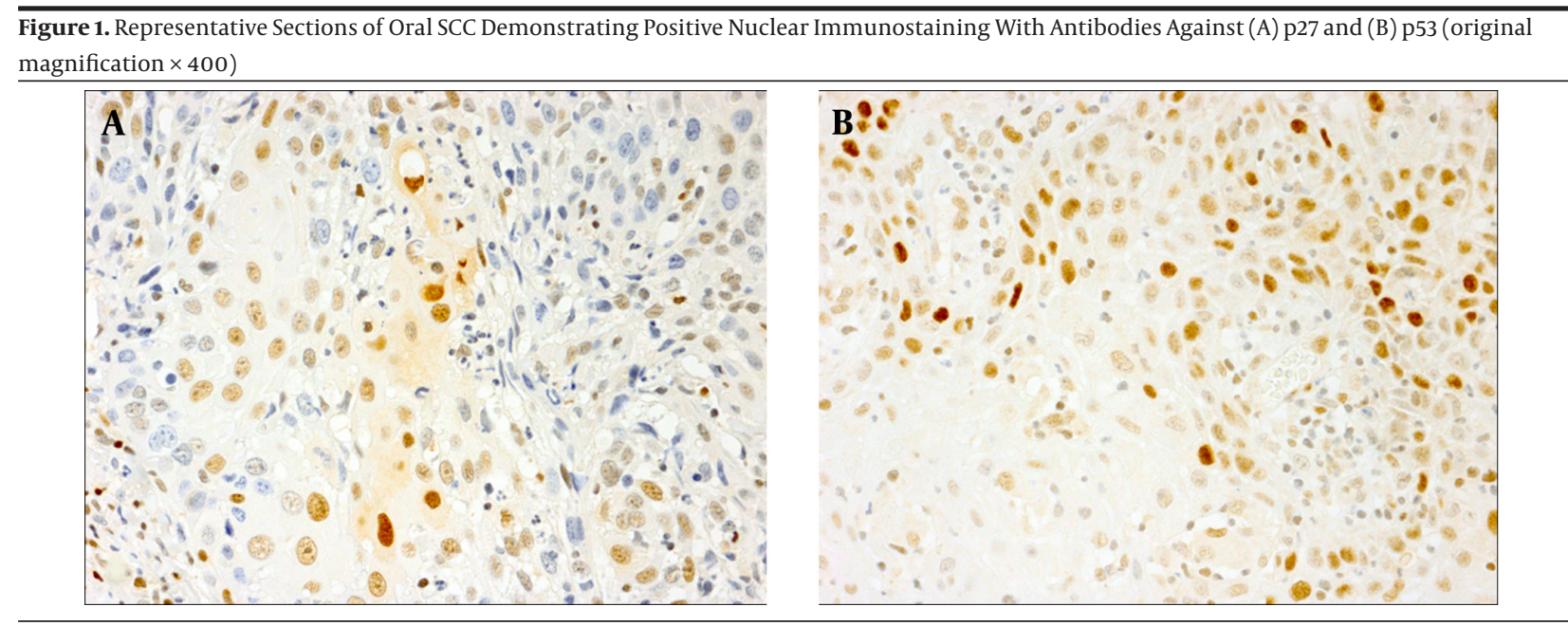

Arrow points to immunostained nuclei of neoplastic cells.

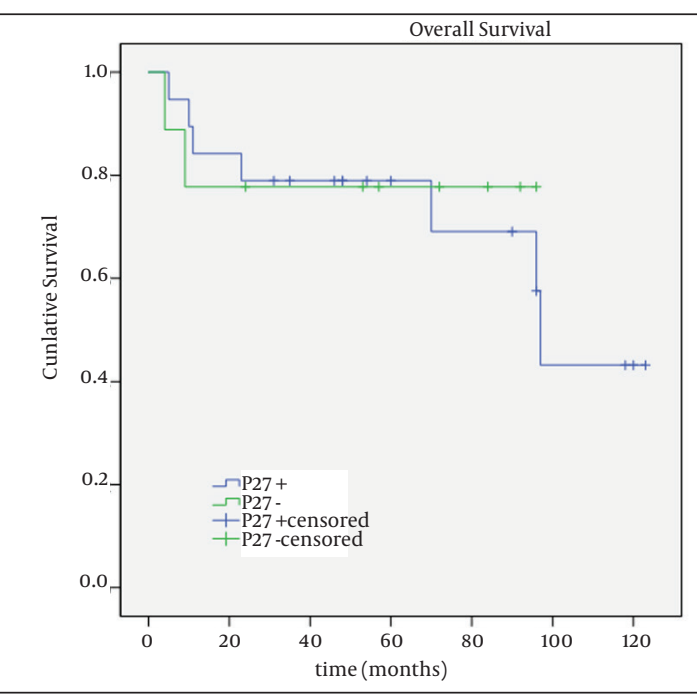

Figure 2. Kaplan-Meier Curve for Overall Survival in p27 Positive and Negative Oral SCC Subjects

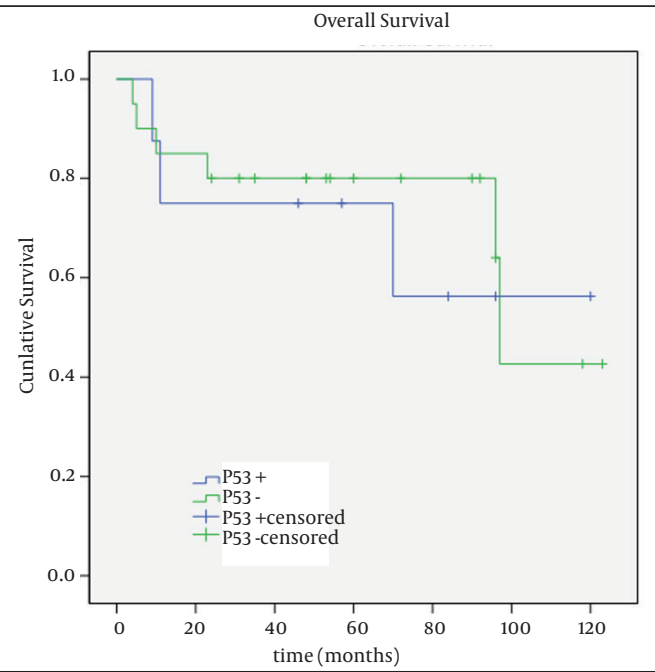

Figure 3. Kaplan-Meier Survival Curves for p53 Positive and Negative Cases in Patients with Oral SCC
Table 1. Univariable Analysis of Overall Survival Based on Clinicopathologic Parameters in Patients With Oral SCC

\begin{tabular}{lcc}
\hline Variables & Number & P Value \\
\hline Age, $y$ & & 0.798 \\
$<50$ & 6 & \\
$>50$ & 22 &
\end{tabular}

\begin{tabular}{|c|c|}
\hline \multicolumn{2}{|l|}{ Gender } \\
\hline Male & 9 \\
\hline Female & 19 \\
\hline
\end{tabular}

\section{Local Recurrence}

0.118

$\begin{array}{ll}\text { Yes } & 10 \\ \text { No } & 18\end{array}$

\section{Tumor grade}

0.048

$\begin{array}{ll}\text { Low + Moderate } & 17 \\ \text { Poor } & 11\end{array}$

$\mathbf{P 2 7}$

0.827

Positive
Negative

\begin{tabular}{lcc} 
P53 & & 0.763 \\
\hline Positive & 8 & \\
\hline Negative & 20 \\
\hline
\end{tabular}




\section{Discussion}

There have been numerous surveys on the role of cell cycle-related factors in the head and neck and oral cavity of cancerous patients from various genetic backgrounds and geographic locations. However, their functional significance and clinical value remain unclear $(9,23)$. In the present study, we evaluated the status of two such factors, p53 and p27, in a subset of Iranian patients and determined their correlation with different clinicopathologic features. Despite the proposition that p27 and p53 may act in concert to induce malignant features like invasiveness and increased proliferation (11, 14, 16, 17), the number of studies simultaneously evaluating these proteins is limited. Furthermore, we were not able to access previous studies on the expression of p27 and p53 in Iranian subjects with oral SCC. Despite the fact that other diagnostic methods like PCR may be more accurate than immunohistochemistry (IHC), it is noteworthy that IHC is more practical and cost efficient in addition to being less technique sensitive. This method is routinely performed in most laboratories and is easily completed on paraffin blocks, which are stored for all patients and retrievable at later dates.

According to our results, there was no significant association between $\mathrm{p} 53$ with either patient survival or tumor recurrence, which was in accordance with a considerable number of former investigations $(6,9,24,25)$. Conversely, there are some studies that have stated a negative effect of p53 expression on prognosis $(7,8,23)$, while Sauter et al. (26) found that expression of this protein prolonged survival.

Immunohistochemistry detects inactivated and stabilized p53 protein, which can result from mutation, binding to products of viruses and cellular oncogenes or 'phosphorylation due to cdc2-like kinases' (6). Immunoreactivity to p53, either because of a functionally altered protein or accumulation of its wild-type form could be indicative of an assault on cellular deoxyribonucleic acid (DNA) or other events, some of which may be in favor of malignant transformation (27). In cells with normally functioning p53, attack on DNA leads to G1 arrest followed by repair, senescence or apoptosis. Alteration of p53 protein could result in loss of its vital actions such as regulating proliferation, inhibiting migration and suppression of invasion. In addition, mutated p53 has been shown to promote metastasis $(4,5,28)$. Therefore, it seems that the trend towards a shorter survival of $\mathrm{p} 53$ positive cases found in the present study is in line with the known function of this protein. There are a considerable number of reasons that might provide an explanation as to why a significant association was not found between p53 expression and survival in the present and previous investigations. Despite the fact that DO-7, used in the current study, has been reported to be an extremely reliable antibody for the detection of p53 protein $(14,24)$, it can also show accumulation of wild-type p53, in which other molecules and pathways are responsible for carcinogenesis. Also the complexity through which mutations affect proteins could help clarify the limited prognostic significance of p53 immunoreactivity. For example, mutations in different codons exert different effects on the function of the protein. In addition nonsense mutations or loss of both alleles can lead to lack of protein detection $(24,29)$.

Tumor grading is considered a traditional prognostic factor (9) with conflicting opinions on its efficiency (30); some favoring its predictive value with high-grade tumors demonstrating worse prognoses $(21,31)$, corroborating our results. It is worth mentioning that the significant relationship between tumor grade and survival found in the present study is marginal and may have been developed by chance. Recent literature has suggested a role of p53 in the control of cellular differentiation via suppression of stem cell genes, but it is unclear whether this function requires developmental signals or it occurs in response to usual stresses $(4,5)$. A significant relationship between $\mathrm{p} 53$ expression and tumor grading was not found in the present study, however our study sample was limited in number and drawing definitive conclusions on this matter would not be wise.

Similar to previous reports $(9,11,14,18,32)$, we did not observe a significant relationship between p27 expression and survival or recurrence. However other studies, including a systematic review conducted by Gao et al. (15), reported shorter survival rates for cases with low p27 expression $(10,12,13)$. This discrepancy may be related to differences in the study population, tumor location, sample size and follow-up periods. It is noteworthy that similar to these investigations, in our study, patients with p27 overexpression were inclined to survive longer than those with a negative p27 status which could be explained by the tumor suppressor and cyclin-dependant kinase (CDK) inhibiting activities of this protein.

According to our results, p53 and p27 were not significantly correlated and their combination did not affect overall survival. The combined status of p53/p27 with or without other markers has been investigated in different tumors and in contrast to our findings significant relationships were found between the two markers $(11,14,16$, 17). On the other hand, studies in the head and neck region, similar to our findings, failed to detect an association between these proteins $(9,18)$. Ozkara and Corakci (11) recognized the involvement of p53 and p27 in cell cycle regulation, but stated that there was no functional relationship between them and considered the two to use different pathways during progression through the cell cycle. However, regarding the role of p27 in spontaneous apoptosis and its correlation with Bax in oral and oropharyngeal SCC, it has been suggested that various components of p53- dependent apoptosis might influence the "p27-related apoptotic pathway" (18). Furthermore, Bai et al. (17) proposed a link between p53 and p27 indicating 
that disruption of the p53 pathway can lead to cyclin E/ CDK2 activation resulting in phosphorylation and subsequent reduction of p27. Therefore, it seems that further studies evaluating the combination of these proteins in head and neck SCC may be helpful in the assessment of the biologic behavior and prognosis of this neoplasm.

In conclusion, according to our results neither p53 nor p27 could be considered prognostic factors in oral SCC; however, lack of prognostic significance does not disprove their possible role in the development of this cancer. Considering the importance of p53 and p27 in oncogenesis and the discrepancy between findings of different studies, unification of laboratory techniques, detection methods and antibodies may be beneficial for future meta-analyses in this field.

\section{Funding/Support}

This research has been supported by cancer research center, cancer institute, Imam Khomeini medical center, Tehran university of medical sciences.

\section{References}

1. Thiel UJ, Feltens R, Adryan B, Gieringer R, Brochhausen C, Schuon $\mathrm{R}$, et al. Analysis of differentially expressed proteins in oral squamous cell carcinoma by MALDI-TOF MS. J Oral Pathol Med. 2011;40(5):369-79.

2. Etemad-Moghadam S, Khalili M, Tirgary F, Alaeddini M. Evaluation of myofibroblasts in oral epithelial dysplasia and squamous cell carcinoma. J Oral Pathol Med. 2009;38(8):639-43.

3. Evan GI, Vousden KH. Proliferation, cell cycle and apoptosis in cancer. Nature. 2001;411(6835):342-8.

4. Stiewe T. The p53 family in differentiation and tumorigenesis. Nat Rev Cancer. 2007;7(3):165-8.

5. Aylon Y, Oren M. New plays in the $\mathrm{p} 53$ theater. Curr Opin Genet Dev. 2011;21(1):86-92.

6. Mineta H, Borg A, Dictor M, Wahlberg P, Akervall J, Wennerberg J. p53 mutation, but not p53 overexpression, correlates with survival in head and neck squamous cell carcinoma. Br J Cancer. 1998;78(8):1084-90.

7. Jalali MM, Heidarzadeh A, Zavarei MJ, Sarmast H. p53 overexpression impacts on the prognosis of laryngeal squamous cell carcinomas. Asian Pac J Cancer Prev. 2011;12(7):1731-4.

8. Kato K, Kawashiri S, Yoshizawa K, Kitahara H, Okamune A, Sugiura $\mathrm{S}$, et al. Expression form of p53 and PCNA at the invasive front in oral squamous cell carcinoma: correlation with clinicopathological features and prognosis. J Oral Pathol Med. 2011;40(9):693-8.

9. Perisanidis C, Perisanidis B, Wrba F, Brandstetter A, El Gazzar S, Papadogeorgakis N, et al. Evaluation of immunohistochemical expression of p53, p21, p27, cyclin D1, and Ki67 in oral and oropharyngeal squamous cell carcinoma. I Oral Pathol Med. 2012;41(1):40-6.

10. Lloyd RV, Erickson LA, Jin L, Kulig E, Qian X, Cheville JC, et al p27kip1: a multifunctional cyclin-dependent kinase inhibitor with prognostic significance in human cancers. Am J Pathol. 1999;154(2):313-23.

11. Ozkara SK, Corakci A. Significantly decreased P27 expression in endometrial carcinoma compared to complex hyperplasia with atypia (correlation with p53 expression). Pathol Oncol Res. 2004;10(2):89-97.

12. Kuo MY, Hsu HY, Kok SH, Kuo RC, Yang H, Hahn LJ, et al. Prognostic role of p27(Kip1) expression in oral squamous cell carcinoma in Taiwan. Oral Oncol. 2002;38(2):172-8.
13. Mineta H, Miura K, Suzuki I, Takebayashi S, Misawa K, Ueda Y, et al p27 expression correlates with prognosis in patients with hypopharyngeal cancer. Anticancer Res. 1999;19(5C):4407-12.

14. Skirnisdottir IA, Sorbe B, Lindborg K, Seidal T. Prognostic impact of p53, p27, and C-MYC on clinicopathological features and outcome in early-stage (FIGO I-II) epithelial ovarian cancer. Int J Gynecol Cancer. 2011;21(2):236-44.

15. Gao L, Gu W, Zheng J, Ren W, Chang S, Wang X, et al. Clinicopathological and prognostic significance of p27 expression in oral squamous cell carcinoma: a meta-analysis. Int J Biol Markers. 2013;28(4):e329-35.

16. Casalini P, Iorio MV, Berno V, Bergamaschi A, Borresen Dale AL, Gasparini P, et al. Relationship between p53 and p27 expression following HER2 signaling. Breast. 2007;16(6):597-605.

17. Bai M, Vlachonikolis J, Agnantis NJ, Tsanou E, Dimou S, Nicolaides $\mathrm{C}$, et al. Low expression of p27 protein combined with altered p53 and $\mathrm{Rb} / \mathrm{p} 16$ expression status is associated with increased expression of cyclin A and cyclin B1 in diffuse large B-cell lymphomas. Mod Pathol. 2001;14(11):1105-13.

18. Fujieda S, Inuzuka M, Tanaka N, Sunaga H, Fan GK, Ito T, et al. Expression of p27 is associated with Bax expression and spontaneous apoptosis in oral and oropharyngeal carcinoma. Int J Cancer 1999;84(3):315-20.

19. Etemad-Moghadam S, Fouladdel S, Azizi E, Alaeddini M. In vitro study on the effect of doxorubicin on the proliferation markers MCM3 and Ki-67. J BUON. 2013;18(4):1062-8.

20. Pinholt EM, Rindum J, Pindborg JJ. Oral cancer: a retrospective study of 100 Danish cases. Br J Oral Maxillofac Surg. 1997;35(2):77-80.

21. Bryne M, Nielsen K, Koppang HS, Dabelsteen E. Reproducibility of two malignancy grading systems with reportedly prognostic value for oral cancer patients. J Oral Pathol Med.1991;20(8):369-72.

22. Carlson ER, Cheung A, Smith B, Pfohl C. Neck dissections for oral/head and neck cancer: 1906-2006. J Oral Maxillofac Surg. 2006;64(1):4-11.

23. Geisler SA, Olshan AF, Weissler MC, Cai J, Funkhouser WK, Smith J, et al. p16 and p53 Protein expression as prognostic indicators of survival and disease recurrence from head and neck cancer. Clin Cancer Res. 2002;8(11):3445-53.

24. Nylander K, Stenling R, Gustafsson H, Zackrisson B, Roos G. p53 expression and cell proliferation in squamous cell carcinomas of the head and neck. Cancer. 1995;75(1):87-93.

25. Frank JL, Bur ME, Garb JL, Kay S, Ware JL, Sismanis A, et al. p53 tumor suppressor oncogene expression in squamous cell carcinoma of the hypopharynx. Cancer.1994;73(1):181-6.

26. Sauter ER, Ridge JA, Gordon J, Eisenberg BL. p53 overexpression correlates with increased survival in patients with squamous carcinoma of the tongue base. Am J Surg.1992;164(6):651-3.

27. Hasegawa M, Ohoka I, Yamazaki K, Hanami K, Sugano I, Nagao T, et al. Expression of p21/WAF-1, status of apoptosis and p53 mutation in esophageal squamous cell carcinoma with HPV infection. Pathol Int. 2002;52(7):442-50.

28. Mountzios G, Rampias T, Psyrri A. The mutational spectrum of squamous-cell carcinoma of the head and neck: targetable genetic events and clinical impact. Ann Oncol.2014;25(10):1889-900.

29. Pim D, Banks L. p53 polymorphic variants at codon $72 \mathrm{ex}$ ert different effects on cell cycle progression. Int $J$ Cancer. 2004;108(2):196-9.

30. de Matos FR, Lima E, Queiroz LM, da Silveira EJ. Analysis of in flammatory infiltrate, perineural invasion, and risk score can indicate concurrent metastasis in squamous cell carcinoma of the tongue. J Oral Maxillofac Surg. 2012;70(7):1703-10.

31. Odell EW, Jani P, Sherriff M, Ahluwalia SM, Hibbert J, Levison DA et al. The prognostic value of individual histologic grading parameters in small lingual squamous cell carcinomas. The importance of the pattern of invasion. Cancer.1994;74(3):789-94.

32. Zhang M, Li J, Wang L, Tian Z, Zhang P, Xu Q, et al. Prognostic significance of p21, p27 and survivin protein expression in patients with oral squamous cell carcinoma. Oncol Lett. 2013;6(2):381-6. 